\title{
Dia Mundial do Rim 2011 Proteinúria e creatinina sérica: testes essenciais para diagnóstico de doença renal crônica
}

\author{
World Kidney Day 2011 \\ Proteinuria and serum creatinine: critical tests for diagnosis chronic kidney disease
}

Gianna Mastroianni Kirsztajn'; Marcus G. Bastos²; Adagmar Andriolo

A doença renal crônica (DRC) é comum e considerada, atualmente, um problema de saúde pública. Isso se deve à constatação do rápido aumento de sua prevalência nos últimos anos, com o agravante de que grande parte dos casos não é diagnosticada nas fases iniciais da doença. Além disso, um aspecto que torna particularmente importante o diagnóstico e o tratamento precoces é que a DRC tem participação relevante no aumento do risco de desenvolvimento de doença cardiovascular $(D C V)^{(3)}$. De fato, é notória a inter-relação entre DRC e DCV, uma favorecendo o aparecimento e complicando a evolução da outra ${ }^{(13)}$. Em ambas as condições, a prevenção pela identificação precoce dos indivíduos em risco e a adoção de bons hábitos de vida se constituem na primeira intervenção a ser realizada.

Em relação à DRC, os casos que se encontram em estágios mais precoces da doença podem ser diagnosticados por testes laboratoriais simples, e o diagnóstico nessa etapa é importante, visto que o tratamento adequado é capaz de reduzir a velocidade de progressão para insuficiência renal crônica em fase avançada, com necessidade de diálise ou transplante, de corrigir as complicações mais frequentes da doença e de prevenir a evolução precoce para o óbito(15).

Deve ser enfatizado que a DRC, em geral, é silenciosa nos estágios iniciais. Assim, o indivíduo acometido pode não apresentar sinais ou sintomas que alertem para a presença de doença, retardando sua detecção, com graves implicações sobre a eficácia dos tratamentos e o prognóstico. É com base nessas observações que se preconiza o uso de testes laboratoriais para o diagnóstico, os quais, idealmente, deveriam ser de baixo custo e amplamente disponíveis na maioria dos laboratórios de análises clínicas.

A dosagem de creatinina, que pode ser utilizada a estimativa da filtração glomerular ( $F G)^{(11)}$, e a pesquisa de proteinúria (albuminúria) são dois testes fundamentais no diagnóstico da DRC. Vale lembrar que, hoje, é amplamente aceita a definição de que é portador de DRC todo indivíduo que apresentar albuminúria e/ ou filtração glomerular estimada (FGe) abaixo de $60 \mathrm{ml} / \mathrm{min} / 1,73 \mathrm{~m}^{2}$, confirmadas em, pelo menos, duas medidas, em um período superior a três meses. Esses testes laboratoriais são reconhecidamente simples, de baixo custo e amplamente disponíveis.

A dosagem de creatinina, na grande maioria dos laboratórios brasileiros, é realizada pela reação de Jaffe, descrita em $1886^{(7)}$. É certo que o procedimento inicialmente descrito foi modificado, viabilizando dosagens diretas no soro, no plasma e na urina. Graças à qualidade dessa análise, a creatinina tornou-se um biomarcador quase universal para a avaliação da $\mathrm{FG}^{(23)}$, sendo possível não só o diagnóstico de doença renal, como o monitoramento preciso da progressão da doença. O desenvolvimento de fórmulas para

1. Professora livre-docente da Universidade Federal de São Paulo (UNIFESP); coordenadora do Ambulatório de Clomerulopatias da UNIFESP; coordenadora da Campanha "Previna-se" e do Comitê de Prevenção de Doença Renal Crônica da Sociedade Brasileira de Nefrologia (SBN).

2. Professor de Nefrologia da Faculdade de Medicina da Universidade Federal de Juiz de Fora (UFFF); coordenador do Núcleo Interdisciplinar de Estudos, Pesquisas e Tratamento em Nefrologia (NIEPEN) da UFJF e da Fundação Instituto Mineiro de Estudos e Pesquisas em Nefrologia (IMEPEN); editor do Jornal Brasileiro de Nefrologia.

3. Médico patologista clínico; professor livre-docente da UNIFESP. 
estimativa da $\mathrm{FG}^{(12)}$ e o estadiamento da doença ${ }^{(17)}$ com base na creatinina sérica evidenciam a importância desse biomarcador. A variação biológica da creatinina é muito pequena, em torno de $4 \%{ }^{(5)}$ e a variação analítica é inferior a 2\%. Evidentemente, há algumas limitações em seu uso. Entre os aspectos laboratoriais, existe a interferência analítica de cromógenos não creatinina, que resistem até aos ensaios cinéticos, e a falta de concordância entre conjuntos diagnósticos de diferentes fabricantes ${ }^{(10)}$. Fisiologicamente, cerca de $28 \%$ da depuração de creatinina em humanos é devida à secreção tubular(21). Drogas que inibem a secreção tubular da creatinina, como salicilatos, fibratos, trimetoprim e cimetidina ${ }^{(18)}$, causam aumento da creatinina sérica independentemente da FG. Contrariamente, drogas como trianterene, espirolactona e amiloride aumentam a secreção tubular renal de creatinina, contribuindo para a redução de sua concentração plasmática. A administração de alguns medicamentos, como hormônio de crescimento e fenofibrato $^{(1)}$, modifica a taxa de produção, em geral aceita como constante.

Apesar dessas limitações, talvez a restrição mais séria tenha sido a evidência apresentada por Shemesh et $a .^{(20)}$ ao demonstrarem que a concentração de creatinina permanece nos intervalos de referência em uma proporção substancial de pacientes com FG altamente comprometida. Esse achado colocou em cheque o valor da creatinina no diagnóstico precoce de doença renal.

Recentemente, Spanaus et al. ${ }^{(22)}$ compararam o desempenho de dois outros biomarcadores de baixo peso molecular, cistatina $C$ e proteína $\beta$-traço, com o da creatinina para diagnóstico, estadiamento e predição de progressão da DRC primária não diabética. A conclusão desse trabalho foi que os três biomarcadores são equivalentes, tanto em termos de desempenho diagnóstico, mesmo em discretos graus de deterioração de função renal, quanto em termos de predição de risco para progressão.

Segundo esses autores, o problema não está propriamente na creatinina, mas em como os resultados dos exames laboratoriais são interpretados, especialmente em relação aos intervalos de referência. A expectativa de que a redução da FG deveria provocar imediatamente níveis de creatinina acima do intervalo de referência é inadequada, mas, considerando a pequena variação biológica e a qualidade analítica atualmente disponível, o monitoramento longitudinal da creatinina permite a detecção precoce do declínio da FG e da doença renal incipiente.

O cálculo da FGe é derivado da creatinina sérica, considerados a idade, o sexo e a raça, os quais, essencialmente, corrigem para massa muscular padrão. Se a massa muscular diferir acentuadamente da média para idade, raça e sexo, a FGe fornecerá resultados menos confiáveis como, por exemplo, os observados nos pacientes amputados e nos desnutridos ${ }^{(11)}$. Nesses indivíduos, a recomendação é determinar a depuração da creatinina em amostra urinária colhida durante 24 horas. Igualmente, não se deve lançar mão da estimativa da FG em crianças, grávidas e indivíduos muito idosos, assim como naqueles cujo peso corporal esteja muito baixo ou muito acima do ideal(9).

Também é preciso ter em mente que a FGe não é acurada o suficiente quando seu valor está acima de $60 \mathrm{ml} / \mathrm{min} / 1,73 \mathrm{~m}^{2}$; a recomendação atual é que os laboratórios que já adotam a prática de estimar a função renal sempre que realizam a dosagem da creatinina sérica não discriminem resultados acima desse valor. É importante lembrar que a creatinina sérica aumenta com a ingestão de carne vermelha cozida, independentemente de alteração da função renal, razão pela qual deveria ser dosada após 12 horas de abstenção de ingestão de carne. De forma semelhante ao que é descrito para a creatinina sérica, a FGe reflete a filtração glomerular somente em estado de equilíbrio, não sendo recomendada, portanto, para estimar a função renal de pacientes com insuficiência renal aguda. A identificação de FGe reduzida impõe a repetição do exame após três meses, com o objetivo de confirmar ou não a cronicidade da alteração funcional renal, ou seja, a existência ou não de DRC ${ }^{(9)}$. 
A pesquisa de proteinúria é válida tanto pela detecção, na urina, da presença de proteína total quanto de albumina. As fitas reagentes, amplamente utilizadas na pesquisa de proteinúria são práticas, mas seu desempenho depende do fluxo de urina e, em geral, não possuem sensibilidade suficiente para a quantificação de microalbuminúria. A creatinina é excretada na urina a uma taxa relativamente constante e pode ser utilizada na parametrização da proteinúria e da albuminúria pelo uso das relações proteína/ creatinina e albumina/creatinina (RAC), respectivamente, em amostras urinárias isoladas. Esses procedimentos apresentam excelente correlação com as determinações realizadas em urina obtida durante 24 horas, além de serem mais confortáveis para o paciente ${ }^{(14)}$.

A RAC apresenta papel central na monitoração da doença renal diabética e deveria ser utilizada, no mínimo, anualmente, tanto no rastreamento quanto no acompanhamento da lesão renal em diabéticos. O papel da relação proteína/creatinina ou da albumina/creatinina em pacientes não diabéticos ainda não está claramente definido nas diretrizes sobre o tema ${ }^{(9,16,19)}$.

A maioria dos estudos sobre desfechos e intervenções baseia-se na determinação da proteinúria total na urina e, menos frequentemente, na albuminúria, cujas pesquisa e dosagem são bem mais dispendiosas, constituindo-se em uma desvantagem. Mais recentemente, alguns autores têm defendido a utilização de um método para detecção de microalbuminúria que utiliza o corante Azul de Coomassie, que teria boa sensibilidade e baixo custo ${ }^{(24)}$.

A classificação da DRC em estágios, atualmente adotada em todo o mundo, baseia-se na FGe e na presença ou não da albuminúria (ou proteinúria). De maneira geral, a incidência de insuficiência renal crônica aumenta com a redução da FGe basal; mas o curso da DRC pode ser diferente, dependendo da presença ou não de alguma evidência de lesão do parênquima renal. De fato, a incidência de insuficiência renal crônica é cerca de 100 vezes maior no paciente que apresenta proteinúria, quando comparada a outro com a mesma $\mathrm{FGe}$, mas sem proteinúria. Em estudo realizado prospectivamente na população holandesa, em uma coorte de 6.879 integrantes, ao longo de 6,2 anos, foi observado que a FGe diminuiu na velocidade de $0,45 \pm 1,60 \mathrm{ml} / \mathrm{min} / 1,73 \mathrm{~m}^{2} /$ ano. Se ajustado para idade e sexo, o declínio da FGe se acentuou gradualmente com o aumento da proteinúria ${ }^{(25)}$. Em qualquer estágio da DRC, a velocidade de queda da FGe foi maior nos indivíduos proteinúricos quando comparados aos não proteinúricos. $\mathrm{O}$ estudo também permitiu identificar hipertensão arterial, glicemia e albuminúria como fatores de risco independentes de progressão da DRC, em ambos os $\operatorname{sexos}^{(6)}$.

Alguns números demonstram, com clareza, a relevância de ser portador de DRC no aumento da probabilidade de morte cardiovascular. Isso fica evidente, por exemplo, quando se observa que um jovem de 20 anos de idade em terapia de substituição renal tem chance de morrer 500 vezes maior que a de um indivíduo saudável, da mesma idade, e é a mesma da de um indivíduo de 80 anos ${ }^{(4)}$. Contudo, essa associação de DRC com mortalidade cardiovascular não se restringe aos pacientes em diálise e pode ser observada naqueles ainda nos estágios pré-dialíticos da doença. É extremamente inquietante saber que a queda da FGe se associa gradualmente à maior mortalidade (principalmente por DCV) e que um paciente com DRC estágio 4 apresenta duas vezes mais chances de óbito do que de evoluir para diálise ${ }^{(8)}$. Contudo, não é só a queda da FGe que se relaciona com maior mortalidade na DRC. Assim, um paciente no estágio 1 ou 2 da DRC (FGe acima de $60 \mathrm{ml} / \mathrm{min} / 1,73 \mathrm{~m}^{2}$ ) e com albuminúria apresenta taxa de mortalidade ajustada duas vezes maior que outro paciente no estágio 3 (FGe de 45-59 ml/min/1,73 m²), mas sem albuminúria(2).

Por fim, a estimativa da FG a partir da creatinina sérica e a determinação da quantidade de proteína ou albumina excretada na urina são testes simples, amplamente disponíveis e de altíssima relevância clínica. 
Deveriam ser realizados regularmente naqueles indivíduos com maior probabilidade de desenvolvimento de DRC (idealmente nos estágios pré-clínicos da doença, quando a FGe é maior que $60 \mathrm{ml} / \mathrm{min} / 1,73 \mathrm{~m}^{2}$ ) e DCV, como os hipertensos e os diabéticos. Quando tais testes são utilizados em conjunto, constituemse em poderosos instrumentos para o diagnóstico de DRC e a definição de prognóstico; além disso, são preditores de desfechos cardiovasculares e de mortalidade.

\section{Referências}

1. ANSQUER, J. C. et al. Effect of fenofibrate on kidney function: a 6-week randomized crossover trial in healthy people. Am J Kidney Dis, v. 51, p. 904-13, 2008.

2. BRENDA, R. et al. Relation between kidney function, proteinuria, and adverse outcomes. JAMA, v. 303, n. 5, p. 423-29, 2010.

3. EKNOYAN, G. et al. The burden of kidney disease: improving global outcomes. Kidney Int, v. 66, n. 4, p. 1310-4, 2004.

4. FOLEY, R. N.; PARFREY, P. S.; SARNAK, M. J. Clinical epidemiology of cardiovascular disease in chronic renal disease. Am J Kidney Dis, v. 32, Suppl 3, p. S112-S119, 1988.

5. GOWANS, E. M.; FRASER, C. G. Biological variation of serum and urine creatinine and creatinine clearance: ramifications for interpretation of results and patient care. Ann Clin Biochem, v. 25, p. 259-63, 1988.

6. HALBESMA, N. et al. PREVEND Study Group: gender differences in predictors of the decline of renal function in the general population. Kidney Int, v. 74, p. 505-12, 2008.

7. JAFFE, M. Ueber den Niederschlag, welchen Pikrinsäure in normalem Harn erzeugt und uber eine Reaction des Kreatinins. Zeitschrift fur Physiologische Chemie, v. 10, p. 391-400, 1886.

8. KEITH, D. S. et al. Longitudinal follow-up and outcomes among a population with chronic kidney disease in a large managed care organization. Arch Intern Med, v. 164, p. 659-63, 2004.

9. KIDNEY Disease Outcomes Quality Initiative. Clinical practice guidelines for chronic kidney disease: evaluation, classification and stratification. Am J Kidney Dis, v. 39, Suppl 1, p. S46-75, 2002.

10. LAMB, E. J. Susceptibility of glomerular filtration rate estimations to variations in creatinine methodology: a study in older patients. Ann Clin Biochem, v. 42, p. 11-8, 2005.

11. LAMB, E. J.; TOMSON, C. R.; RODERICK, P. J.; Clinical Sciences Reviews Committee of the Association for Clinical Biochemistry. Estimating kidney function in adults using formulae. Ann Clin Biochem, v. 42, Pt 5, p. 321-45, 2005.

12. LEVEY, A. S. et al. Expressing the modification of diet in renal disease study equation for estimating glomerular filtration rate with standardized serum creatinine values. Clin Chem, v. 53, p. 766-72, 2007.

13. MASTROIANNI KIRSZTAJN, G. Renal function markers as prevention tools. J Bras Nefrol, v. 28, Suppl 2, p. 48-52, 2006.

14. McINTYRE N. J.; TAAL, M. W. How to measure proteinuria? Curr Opin Nephrol Hypertens, v. 17, p. 600-3, 2008.

15. MIDDLETON, J. P.; PUN, P. H. Hypertension, chronic kidney disease, and the development of cardiovascular risk: a joint primacy. Kidney Int, v. 77, p. 753-5, 2010.

16. NATIONAL Institute for Health and Clinical Excellence. Chronic kidney disease. Early identification and management of chronic kidney disease in adults in primary and secondary care. Clinical guideline CG73. London: NICE, 2008. Disponível em: <http://www.nice.org.uk/Guidance/CG73>. Acesso em: 22 dez. 2010.

17. NATIONAL Kidney Foundation. KIDOQI clinical practice guidelines for chronic kidney disease: evaluation, classification, and stratification. Am J Kidney Dis, v. 39, Suppl 1, p. S1-266, 2002.

18. PREUSS, H. G. Organic cationic drugs and renal creatinine secretion. Ren Physiol, v. 6, p. 103, 1983.

19. SCOTTISH Intercollegiate Guideline Network. Diagnosis and management of chronic kidney disease. A national clinical guideline. SIGN Guideline 103. Edinburgh: SIGN, 2008. Disponível em: <http://www.sign.ac.uk/pdf/sign103. pdf $>$. Acesso em: 22 dez. 2010.

20. SHEMESH, O. et al. Limitations of creatinine as a filtration marker in glomerulopathic patients. Kidney Int, v. 28, p. 830-8, 1985.

21. SMITH, H. W. The kidney: structure and function in health and disease. New York: Oxford University Press, 1951. p. 63-6.

22. SPANAUS, K-S. et al. Serum creatinine, cystatin $C$, and $\beta$-trace protein in diagnostic stagin and predicting progression of primary nondiabetic chronic kidney disease. Clin Chem, v. 56, p. 740-9, 2010.

23. SPENCER, K. Analytical reviews in clinical biochemistry: the estimation of creatinine. Ann Clin Biochem, v. 23, p. 1-25, 1984.

24. TUGIRIMANA, P. L.; DELANGHE, J. R. Development of an affordable dye-stained microalbuminuria screening test. Nephrol Dial Transplant, v. 24, p. 1485-90, 2009.

25. van der Velde, M. Screening for albuminuria identify subjects at increased renal risk. J Am Soc Nephrol, v. 20, n. 4, p. 852-62, 2009. 